\title{
Werden wir einst gegen unseren Willen behandelt?
}

\author{
Hans Stalder \\ Prof. Dr. med., Mitglied der Redaktion
}

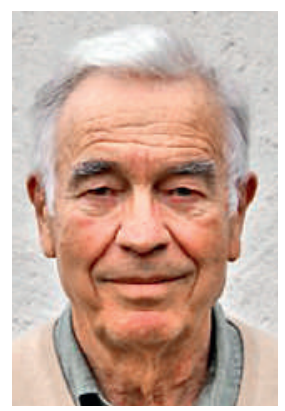

Ältere Menschen bringen oft zum Ausdruck, dass sie lieber sterben möchten, als dement zu werden. In einer britischen Studie wünschten sich 60\% der Befragten [1] bei Demenz Zugang zu aktiver Sterbehilfe und in Belgien lag dieser Prozentsatz bei über 71\% [2]. Warum kann diesem Wunsch nicht stattgegeben werden? Die persönliche Autonomie wäre gewährleistet, denn der Wunsch wurde frei zum Ausdruck gebracht und der Person wird somit nicht geschadet. Und aus einer aktiven Sterbehilfe erwächst keinerlei Belastung für die Gesellschaft, im Gegenteil: Häufig möchten die Betroffenen weder den Angehörigen noch den Pflegenden zur Last fallen. Ein solches Vorgehen ist in der Schweiz ausgeschlossen, denn die aktive Sterbehilfe gilt als Tötung und daher als strafbar, selbst wenn sie aus ehrenwerten Motiven namentlich aus Mitleid - geschieht (Art. 114 StGB). Der assistierte Suizid wird in der Schweiz nur dann strafrechtlich verfolgt, wenn selbstsüchtige Beweggründe vorliegen. Dabei muss der Patient urteilsfähig sein, was im Falle einer fortgeschrittenen Demenz natürlich nicht gewährleistet ist. Daher hat Exit dieses Vorgehen in einer solchen Situation klar zurückgewiesen. In den Niederlanden ist die aktive Sterbehilfe seit 2002 auch bei Demenz zulässig, wenn eine klare schriftliche Patientenverfügung dazu vorliegt (in Belgien wurde 2015 ein ähnlicher Gesetzesvorschlag eingebracht [2]), so dass anzunehmen wäre, dass die Sterbehilfe dort bei Demenz auch ausgeführt wird. Tatsache ist jedoch, dass in den 2200 Fällen, in denen in vorliegenden Patientenverfügungen explizit nach aktiver Sterbehilfe bei Demenz verlangt worden war, keine einzige realisiert wurde [3]. Dafür lassen sich zwei Gründe anführen: In der niederländischen Gesetzgebung zur Sterbehilfe wird u.a. verlangt, dass «unerträgliches, hoffnungsloses Leiden» vorliegt. Aus Sicht der Pflegenden mag bei Demenzpatienten zwar unerträgliches Leiden gegeben sein, doch ohne echten Dialog lässt sich unmöglich feststellen, ob dies für den Patienten tatsächlich auch der Fall ist. Selbst wenn die Pflegenden oder die Angehörigen dies so empfinden, verlangt das Gesetz, dass «Arzt und Patient zu der Überzeugung gelangt sein müssen, dass es keine anderen Perspektiven gibt». Wie aber kann Sicherheit darüber erlangt werden? Vielleicht hat der an Demenz erkrankte Pa- tient seine Meinung geändert und kann dies nicht zum Ausdruck bringen. Dieser Mangel an Kommunikation - Letztere eine unabdingbare Voraussetzung in einer so kritischen Situation - zwischen Arzt und Patient führt dazu, dass die Ärzte, selbst wenn sie einer aktiven Sterbehilfe unter anderen Umständen positiv gegenüberstehen, diese bei dementen Patienten nie durchgeführt haben [4].

Es folgt daraus, dass selbst wenn die meisten Menschen im Falle einer Demenz den Tod vorgezogen hätten, einem solchen Verlangen offenbar nicht stattgegeben werden kann. Wenn wir die Daten aus Grossbritannien und Belgien [1, 2] auf die Schweiz übertragen, würden zwischen 60\% und 70\% der etwa 120000 an Demenz erkrankten Personen, das heisst mehr als 70000 , gegen ihren Willen behandelt ...

Jenen Menschen, denen diese Situation unerträglich erscheint, bleibt immer noch der Rückgriff auf den assistierten Suizid in der Zeit zwischen dem Auftreten einer beginnenden Demenz, der mild cognitive impairment (MCI), wenn die Urteilsfähigkeit noch gegeben ist, und dem Zustand der vollständigen Urteilsunfähigkeit. Einigen Studien [5] zufolge liegt jedoch die Wahrscheinlichkeit einer tatsächlich eintretenden Demenz nach MCI-Diagnose bei nur 5\% pro Jahr (im Schnitt etwa 10\%) und manchmal verbessert sich das Gedächtnis bei MCIPatienten. Wer möchte denn schon sterben, wenn gute Chancen bestehen, noch eine Weile ohne Demenz leben zu können?

Dies ist für mich ein unlösbarer Konflikt - aber vielleicht haben Sie, liebe Leserinnen und Leser, eine Lösung?

\section{Literatur}

1 Williams N, Dunford C, Knowles A, Warner J. Public attitudes to life-sustaining treatments and euthanasia in dementia. Int J Geriatr Psychiatry. 2007;22:1229-34.

2 Belgische Abgeordnetenkammer. Gesetzesvorlage 1013/001. https://www.lachambre.be/

3 Rurup ML, Onwuteaka-Philipsen BD, van der Heide A, van der Wal G, van der Maas PJ. Physicians' experiences with demented patients with advance euthanasia directives in the Netherlands. J Am Geriatr Soc. 2005;53:1138-44.

4 Bolt EE, Snijdewind MC, Willems DL, van der Heide A, Onwuteaka-Philipsen BD. Can physicians conceive of performing euthanasia in case of psychiatric disease, dementia or being tired of living? J Med Ethics. 2015;41:592-8.

5 Langa KM, Levine DA. The diagnosis and management of mild cognitive impairment: a clinical review. JAMA. 2014;312:2551-61. 